\title{
A Preliminary Study of the Objective Measurement of Compliance Rates for Semirigid Lumbar-Support Use in Patients with Chronic Nonspecific Low Back Pain: How Important Is the Compliance Rate?
}

\author{
Maryam Hekmatfard ${ }^{1}$, Mohammad Ali Sanjari ${ }^{2}$, Nader Maroufi, \\ Hassan Saeedi ${ }^{1}$, Esmail Ebrahimi ${ }^{3}$, Hamid Behtash ${ }^{4}$ \\ ${ }^{1}$ Department of Orthotics and Prosthetics, School of Rehabilitation Sciences, Iran University of Medical Sciences, Tehran, Iran \\ ${ }^{2}$ Department of Rehabilitation Basic Sciences, School of Rehabilitation Sciences, and Biomechanics Lab., \\ Rehabilitation Research Center, Iran University of Medical Sciences, Tehran, Iran \\ ${ }^{3}$ Department of Physiotherapy, School of Rehabilitation Sciences, Iran University of Medical Sciences, Tehran, Iran \\ ${ }^{4}$ Department of Orthopedic and Spinal Surgery, School of Medicine, Iran University of Medical Sciences, Tehran, Iran
}

\section{Study Design: Clinical pilot study.}

Purpose: To objectively evaluate the compliance rate of lumbar-support use in patients with chronic nonspecific low back pain, as well as to assess low back pain intensity, disability, and fear-avoidance beliefs.

Overview of Literature: Wearing time is an important factor in the assessment of the efficacy of lumbar-support use in patients with chronic nonspecific low back pain. Previous studies have measured lumbar-support wearing time based on subjective assessment, and these evaluations are not easily verifiable and are usually overestimated by subjects.

Methods: Twelve subjects with chronic nonspecific low back pain who had been wearing semirigid lumbar supports for 6 weeks were evaluated. Compliance was objectively monitored using temperature sensors integrated into the semirigid lumbar supports. Subjects wore their lumbar supports for 8 hour/day on workdays and 3 hour/day on holidays during the first 3 weeks. During the next 3 weeks, subjects were gradually weaned off the lumbar supports. Pain intensity was measured using a numerical rating scale. The Oswestry disability index was used to assess the subjects' disability. Fear-avoidance behavior was evaluated using a fear-avoidance beliefs questionnaire.

Results: The mean compliance rate of the subjects was $78.16 \% \pm 13.9 \%$. Pain intensity was significantly lower in patients with a higher compliance rate $(p=0.001)$. Disability index and fear-avoidance beliefs (functional outcomes) significantly improved during the second 3-weeks period of the treatment $(p<0.001, p=0.02$, respectively).

Conclusions: The compliance rate of patients wearing lumbar supports is a determining factor in chronic low back pain management. Wearing semirigid lumbar supports, as advised, was associated with decreased pain intensity, improved disability index scores, and improved fear-avoidance beliefs in patients with chronic nonspecific low back pain.

Keywords: Chronic nonspecific low back pain; Lumbar supports; Compliance; Wearing time; Temperature logger 


\section{Introduction}

Low back pain (LBP) is one of the most common musculoskeletal disorders. The lifetime prevalence of LBP is approximately $70 \%-85 \%$ [1]. The recurrence rate of LBP is as high as $44 \%-78 \%$, which can lead to increased absenteeism from work [2]. Chronic back pain imposes high costs on the healthcare system, and back pain has been receiving increasing prominence in the literature. Pain management for chronic LBP includes various treatment strategies. Rehabilitation, as a nonoperative treatment modality, plays an important role in chronic LBP management. Rehabilitation protocols employ lumbar supports for prevention and treatment of chronic LBP. The efficiency of lumbar supports is in doubt. The most important factor influencing the effectiveness of lumbar supports is whether patients wear the lumbar supports for a sufficient duration as recommended [3].

Previous studies have investigated the wearing time of prescribed lumbar supports or the compliance rate according to subjective questionnaires [4-10]. Although Kawchuk et al. [11] attempted to use a data logger for determining the compliance rate in such patients, the custom-made sensors that they integrated in the supports failed to monitor lumbar-support wearing time during the intervention period. Compliance rate was reported as follows: a total of $43 \%$ participants wore lumbar supports for at least half the time, and $80 \%$ of them wore lumbar supports most of the time [6]. In addition, subjects who wore the lumbar supports at work for prevention purposes were less compliant $[4,5]$. In another study, $56 \%$ subjects wore the lumbar supports $>7$ hours a day [12].

Past studies have focused on subjective statements on lumbar-support wearing time [4-10]. One of the major drawbacks of subjective statements is the difficulty in verifying the subjects' statements. Moreover, subjects usually overestimate their lumbar-support wearing time [13].

The aim of the present study was to objectively evaluate the compliance rate of lumbar-support use in patients with chronic nonspecific LBP. The current study was also designed to determine how essential is to monitor this compliance rate is for lumbar-support use.

\section{Materials and Methods}

\section{Participants}

Twelve subjects with chronic nonspecific LBP participated in this clinical pilot study (Table 1). Subjects suffering from detectable spine and lower limb injuries, and those who had previously used lumbar supports were excluded. In all subjects, LBP persisted for $>12$ weeks [2]. They had several recurrent episodes of LBP. Even if the subjects were referred to clinics, they were required to have an

Table 1. Characteristics of subjects

\begin{tabular}{lcccc} 
& & \multicolumn{2}{c}{ Demographic data } \\
\cline { 2 - 5 } Subjects & Age $(\mathrm{yr})$ & Sex & BMI $\left(\mathrm{kg} / \mathrm{m}^{2}\right)$ & Pain chronicity $(\mathrm{yr})$ \\
\hline 1 & 27 & Male & 26.60 & 6 \\
\hline 2 & 44 & Female & 26.70 & 9 \\
\hline 3 & 36 & Female & 25.09 & 6 \\
\hline 4 & 28 & Female & 23.93 & 7 \\
\hline 5 & 35 & Male & 25.30 & 3 \\
\hline 6 & 50 & Female & 26.34 & 20 \\
\hline 7 & 20 & Female & 24.84 & 1 \\
\hline 8 & 24 & Female & 20.07 & 4 \\
\hline 9 & 32 & Female & 29.61 & 2 \\
\hline 10 & 20 & Female & 21.56 & 0.5 \\
\hline 12 & 30 & Female & 22.77 & 1 \\
\hline Mean & 31 & Male & 24.63 & 5 \\
\hline BSD & $31.42 \pm 8.98$ & $24.79 \pm 2.52$ & $5.38 \pm 5.31$ \\
\hline
\end{tabular}

BMI, body mass index; SD, standard deviation. 
acute onset of LBP.

The process of the study was described to the subjects, and all signed an informed consent form approved by the Medical Ethics Committee of Iran University of Medical Sciences.

\section{Study setting and intervention}

All subjects wore semirigid nonelastic lumbosacral orthosis (LSO, Exos Form, DJO Global LLC., Vista, CA, USA) for 6 weeks (Fig. 1). The protocol for wearing LSO was as follows: subjects were instructed to wear the lumbar supports for 8 hour/day during workdays and 3 hour/day during holidays for the first 3 weeks. They were gradually weaned off their LSO during the second 3 weeks, such that wearing hours decreased to 6.5 hours during the fourth week, 5 hours during the fifth week, and 4 hours during the sixth week during workdays. During holidays, subjects wore LSO for 2 hours over the second 3 weeks. This protocol was explained to the subjects using both written and verbal instructions. They were instructed on the technique of wearing LSO and tightening it around their lumbar areas. The LSO tightening mechanism provided independent superior and inferior compression to ensure an optimal fit.

LBP intensity was measured using a numerical rating scale. This scale is an 11-point scale from 0 through 10; 0 representing "no pain" and 10 representing "worst imaginable pain" $[14,15]$. Subjects were instructed to select a number that best matched their pain intensity. In our study, the pain intensity was recorded at the end of the first 3 weeks, and at the ends of the fourth, fifth, and sixth weeks and at 1-month follow-up after cessation of wearing LSO.

Functional outcomes of the subjects were assessed using the Oswestry disability index (ODI) $[16,17]$, a self-report

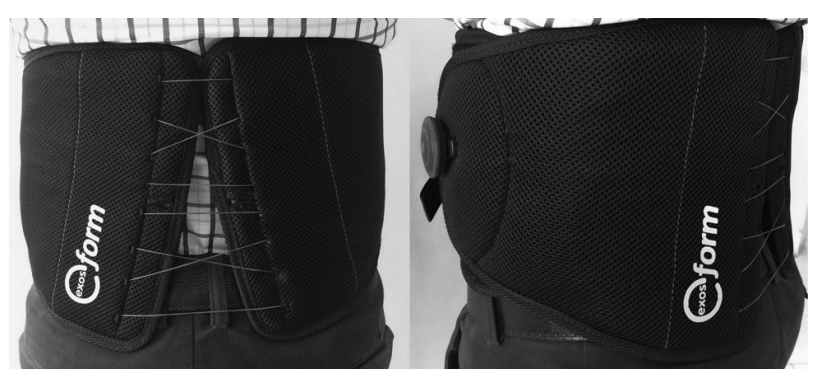

Fig. 1. Posterior and lateral view of the semirigid lumbosacral orthosis. questionnaire that assesses functional disabilities due to LBP. ODI consists of 10 sections, and each section has six statements. Subjects were asked to select one statement from each section that best described their situation. Each section was scored from 0 to 5 ; the scores were added, divided by the number of total responded sections (50 if all sections were responded) and multiplied by 100. A score of 0 represented no disability, and a score of 100 represented complete disability.

The fear-avoidance behavior of subjects was evaluated using the fear-avoidance beliefs questionnaire (FABQ) $[18,19]$. FABQ contains 16 items and two sections related to work and physical activity. Each item is scored from 0 to 6 , so that a higher score represents a high level of fearavoidance beliefs. We calculated the scores of four items from the physical activity section and seven items from the work section. A total overall score ranged from 0 to 66 , with higher scores denoting higher fear-avoidance beliefs. Subjects answered the ODI and FABQ questionnaires at the end of the first 3 weeks, at the end of the fourth, fifth, and sixth weeks, and at 1-month follow-up after the cessation of wearing LSO.

All subjects were prescribed meloxicam $7.5 \mathrm{mg}$ orally once daily for 2 days. The subjects were not often interested in pain relievers.

\section{Objective measurement of compliance}

A temperature logger was used to monitor actual LSO wearing time. The wearing time measurement system (Orthotimer, Rollerwerk Medical Engineering and Consulting, Balingen, Germany) has a small microsensor, including a battery $(13 \times 9 \times 4.5 \mathrm{~mm})$, which is easily inte-

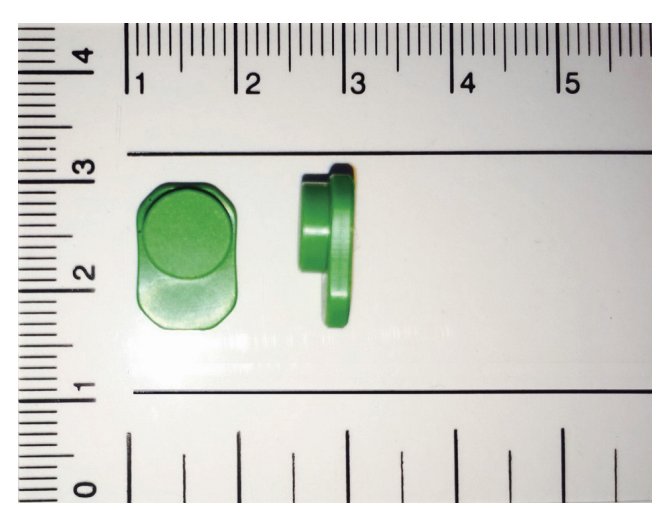

Fig. 2. The temperature logger. Front and side view of microsensor (included battery). 
grated into the anterior part of LSO (Fig. 2). Subjects were not informed of the sensor to avoid bias. The microsensor measures and stores the time, date, and temperature every 15 minutes. Each sensor has a unique identification number that enables it to connect automatically to the patient's records in the database. The saved data transfers wirelessly via a reading device in the corresponding software. The temperature threshold was taken as $28^{\circ} \mathrm{C}$. Temperatures between $28^{\circ} \mathrm{C}$ and $32^{\circ} \mathrm{C}$ indicate the time intervals that subjects wore their LSO [13,20-22]. However, ambient temperature may influence the sensor, which can result in a false-positive report of wearing time. To detect such spurious recordings, data was inspected to exclude continuously recorded values of around $\geq 28^{\circ} \mathrm{C}$ in 24 -hour intervals. The invalid periods, indicating the periods when LSO may not have been actually worn, were excluded from the wearing time report [23]. It was also assumed that LSO is not worn during night time.

Compliance rate was determined as the ratio of LSO wearing time monitored by the microsensor to the recommended LSO wearing time multiplied by one hundred.

To measure the compliance, we considered periods as recommended for the wearing time during the 6 weeks. That is, the compliance rate was reported at the end of the first 3 weeks and at the end of the fourth, fifth, and sixth weeks (Table 2). The least wear time to consider the subjects compliant was set at 3 hour/week.

Table 2. Wearing time (hours) of the LSO during 6-week intervention period

\begin{tabular}{|c|c|c|c|c|}
\hline \multirow{2}{*}{ Time } & \multirow{2}{*}{ First 3 wk } & \multicolumn{3}{|c|}{ Second 3 wk } \\
\hline & & 4th wk & 5th wk & 6th wk \\
\hline Workdays (hr) & 8 & 6.5 & 5 & 4 \\
\hline Holidays (hr) & 3 & 2 & 2 & 2 \\
\hline
\end{tabular}

LSO, lumbosacral orthosis.

\section{Statistical analysis}

For statistical analysis of data, a one-way analysis of variance (ANOVA) design was used to compare differences in pain intensity, disability scale, and fear-avoidance behavior among subjects, as well as the compliance rate between subgroups. The Pearson correlation coefficient was employed to investigate the relationship between compliance rate and pain intensity, disability scale, and fear-avoidance behavior. A one-way repeated measures ANOVA design was used to compare differences in pain intensity, disability scale, and fear-avoidance behavior and compliance rate during the 6 weeks wearing the LSO.

\section{Results}

\section{Compliance rate}

The mean compliance rate was $78.16 \%( \pm 13.9 \%)$ with a range from $58.83 \%$ to $100 \%$. Fifty percent of subjects had compliance rate of $>80 \%$. Table 3 shows the mean compliance rate, pain intensity, and functional outcomes during the 6-week treatment period and at 1-month follow-up.

\section{Compliance rate and pain intensity}

The relationship between compliance rate and pain intensity during 6-week treatment was investigated using a Pearson correlation coefficient. There was a strong and negative correlation $(r=-0.491, p<0.001)$ with high levels of compliance rate and lower levels of pain intensity.

A one-way repeated measure ANOVA design was conducted to compare the pain intensity before the intervention with 6-week treatment interval and 1-month followup after treatment (Table 3). Fig. 3 shows the pain intensity significantly decreased during the 6-week treatment period

Table 3. Compliance rate, pain intensity, and functional outcomes during 6-week intervention period and 1-month follow-up

\begin{tabular}{|c|c|c|c|c|c|c|c|}
\hline \multirow{2}{*}{ Variables } & \multirow{2}{*}{$\begin{array}{c}\text { Baseline } \\
\text { measurement }\end{array}$} & \multicolumn{5}{|c|}{ 6-week Intervention period } & \multirow{2}{*}{$\begin{array}{l}\text { 1-month } \\
\text { Follow-up }\end{array}$} \\
\hline & & First 3 wk & 4th wk & 5th wk & 6th wk & Total (6 wk) & \\
\hline Compliance rate (\%) & - & $71.9 \pm 20.19$ & $70.82 \pm 24.9$ & $81.62 \pm 18.67$ & $88.25 \pm 18.71$ & $78.16 \pm 13.9$ & - \\
\hline Pain intensity & $5.33 \pm 1.06$ & $2.00 \pm 1.42$ & $1.25 \pm 1.29$ & $1.25 \pm 1.29$ & $1.33 \pm 1.38$ & $1.46 \pm 1.34$ & $1.17 \pm 1.4$ \\
\hline Oswestry disability index score & $24.67 \pm 10.95$ & $17.17 \pm 9.52$ & $10.37 \pm 7.73$ & $10.37 \pm 7.73$ & $10.37 \pm 7.73$ & $12.07 \pm 8.48$ & $8.67 \pm 2.3$ \\
\hline Fear avoidance beliefs score & $31.33 \pm 11.9$ & $21.17 \pm 10.43$ & $19.00 \pm 11.57$ & $19.00 \pm 11.57$ & $19.00 \pm 11.57$ & $20.98 \pm 11.48$ & $16.83 \pm 10.57$ \\
\hline
\end{tabular}

Values are presented as mean \pm standard deviation. 
and 1-month after cessation of wearing LSO $(p<0.001)$.

\section{Compliance rate, disability, and FABQ index}

The relationship between the compliance rate and the disability and the FABQ index of subjects during the 6-week treatment was investigated using a Pearson correlation

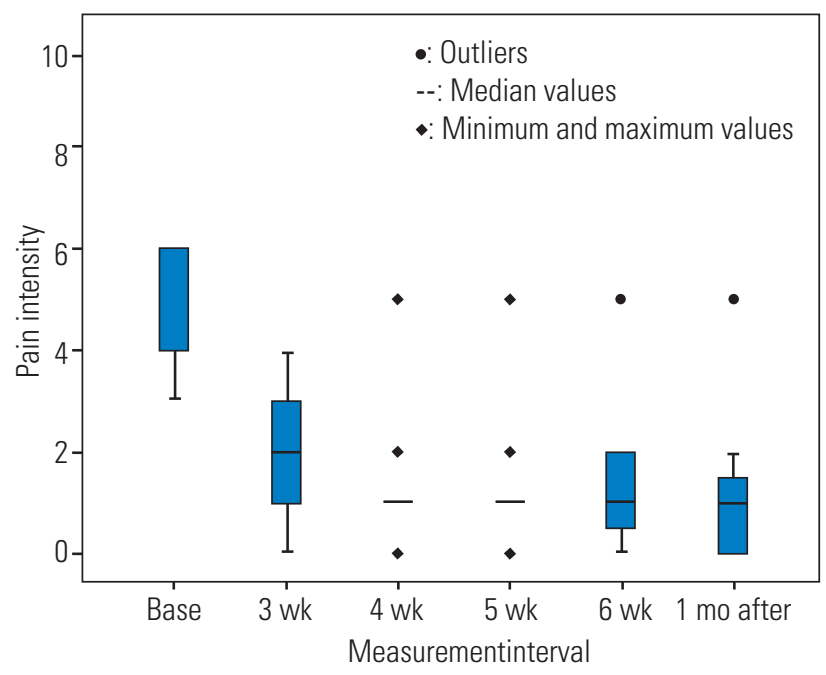

Fig. 3. Trends for pain intensity during the 6-week intervention period and 1-month follow-up. There is a significant difference between baseline measurement and other measurement intervals. Base, baseline measurement; 3 wk, first 3 weeks; 4 wk, 5wk, 6 wk, 4th, 5th, 6th week; 1 mo after, 1 month follow-up.

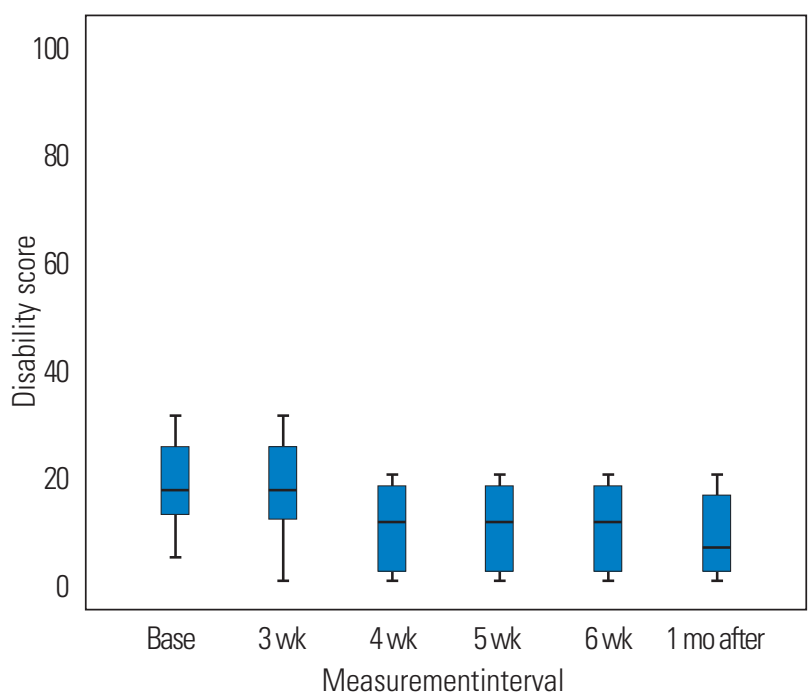

Fig. 4. Trends for the disability score during 6-week intervention period and 1-month follow-up. There is a significant difference between baseline measurement and 4th-week, 5th-week, 6th-week, and 1-month follow-up measurements.Base, baseline measurement; 3 wk, first 3 weeks; 4 wk, 5wk, 6 wk, 4th, 5th, 6th week; 1 mo after, 1 month follow-up. coefficient. There was no significant correlation $(r=0.036$, $p=0.81, r=0.198, p=0.19$, respectively) between compliance rate, disability and the FABQ index of subjects.

A comparison between disability scale and FABQ index of subjects before the intervention and at 6-week treatment interval and 1-month follow-up after treatment showed that the disability scale and the FABQ index of subjects significantly decreased during the second 3 weeks (from the fourth to the sixth week) of wearing the LSO and after 1-month follow-up ( $p=0.001, p=0.03$, respectively) (Figs. 4, 5).

\section{Comparing the results of compliance}

All subjects were categorized into three different groups according to their compliance rates (low: compliance $<60 \%$, medium: $60 \% \leq$ compliance $\leq 99 \%$, and high: compliance $=100 \%$, Table 4 ). There was statistical significant difference in pain intensity between the three different groups (F $[2,45]=7.65, p=0.001$ ). Post-hoc comparisons using the Tukey honestly significant difference (HSD) test indicated that the pain intensity was significantly lower in subjects who had high compliance rate compared to subjects who had a low compliance rate (Fig. 6). There was no significant difference in the disability and fearavoidance beliefs between three categories of compliance

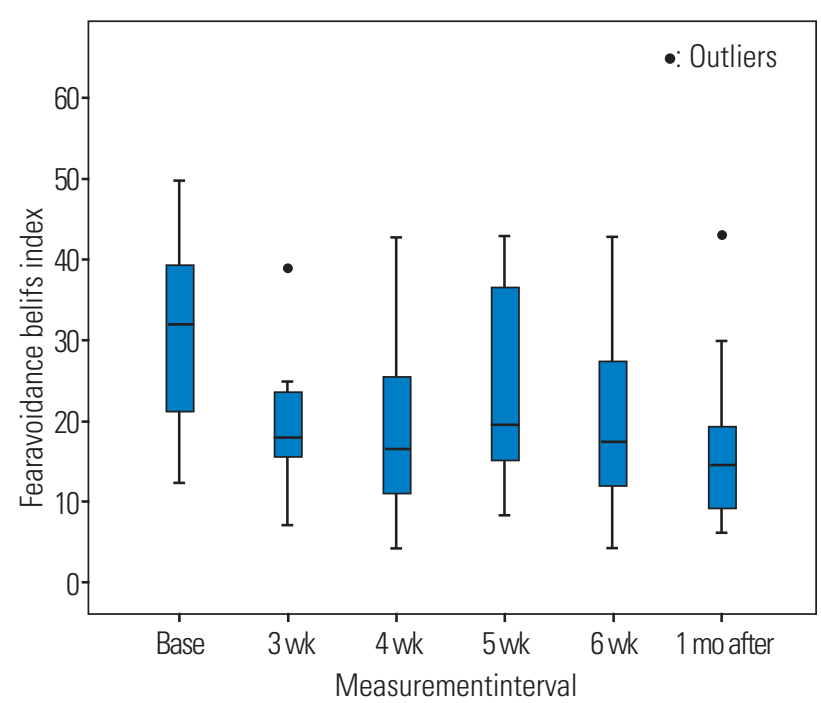

Fig. 5. Trends for the fear-avoidance beliefs index during 6-week intervention period and 1-month follow-up. There is a significant difference between baseline measurement and 4th-week, 5th-week, 6th-week and 1-month follow-up measurements. Base, baseline measurement; 3 wk, first 3 weeks; 4 wk, 5wk, 6 wk, 4th, 5th, 6th week; 1 mo after, 1 month follow-up. 
Table 4. Pain intensity and functional outcomes of subjects according to the compliance rate

\begin{tabular}{|c|c|c|c|c|}
\hline Variables & Compliance $<60 \%$ & $60 \% \leq$ Compliance $\leq 99 \%$ & Compliance $=100 \%$ & $p$-value \\
\hline Pain intensity & $2.37 \pm 1.71$ & $1.18 \pm 0.9$ & $0.8 \pm 0.66$ & 0.001 \\
\hline Oswestry disability index scale & $12.85 \pm 8.64$ & $10.39 \pm 8.8$ & $12.96 \pm 8.32$ & 0.63 \\
\hline Fear avoidance beliefs index & $19.19 \pm 11.1$ & $18.44 \pm 11.54$ & $25.3 \pm 10.3$ & 0.18 \\
\hline
\end{tabular}

Values are presented as mean \pm standard deviation.

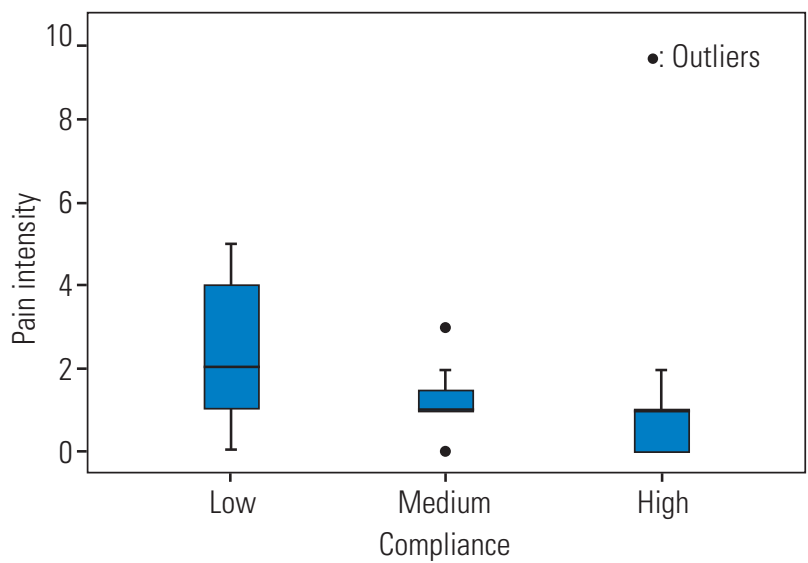

Fig. 6. Pain intensity according to the compliance rates. There is a significant difference between the pain intensity in low compliance and two other groups. Low, compliance $<60 \%$; Medium, $60 \% \leq$ compliance $\leq 99 \%$; High, compliance $=100 \%$.

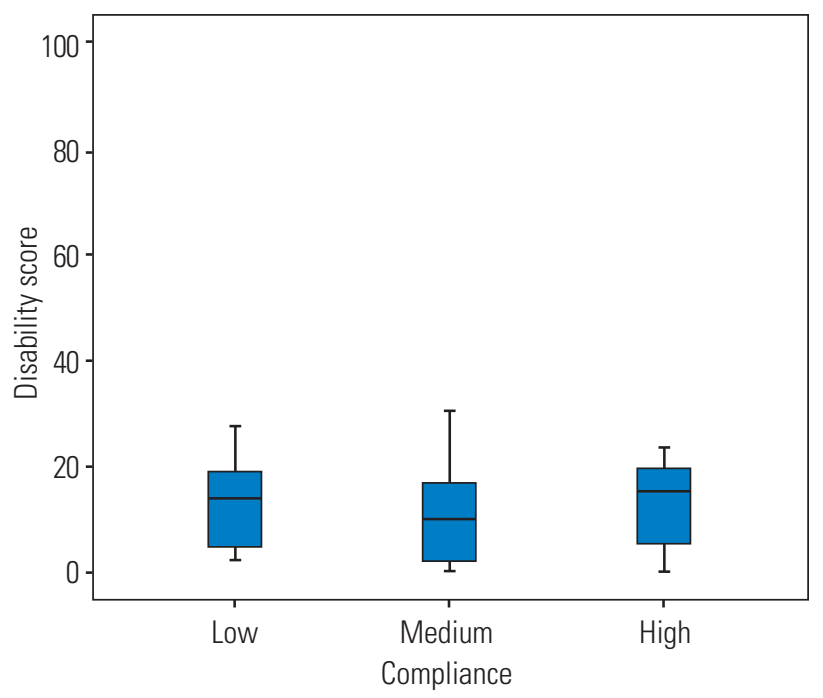

Fig. 7. Disability score according to compliance rates. Low, compliance $<60 \%$, Medium, 60\% $\leq$ compliance $\leq 99 \%$; High, compliance $=100 \%$.

rates ( $p=0.63, p=0.18$, respectively) (Figs. 7,8$)$.

\section{Discussion}

This is the first study to involve objective monitoring of lumbar-support wearing time in patients with chronic

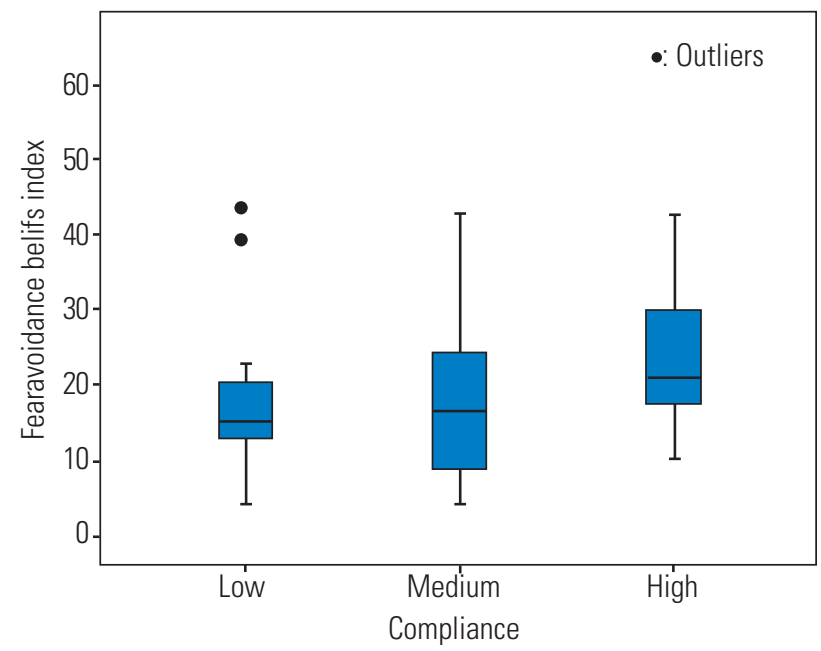

Fig. 8. Fear-avoidance beliefs index according to compliance rates. Low, compliance $<60 \%$; Medium, 60\% $\leq$ compliance $\leq 99 \%$; High; compliance $=100 \%$.

nonspecific LBP. Lumbar-support wearing time was measured by reliable temperature-recording sensors, which were integrated into LSO. The average compliance rate was approximately $78 \%$, with $50 \%$ subjects having a rate of approximately $80 \%$, which reveals that the subjects were well compliant. In comparison with other studies that assessed lumbar-support wearing time according to subjects' statements, our findings are similar to those of Morrisette et al. [9] and roughly consistent with the findings of the study conducted by Hsieh et al. [12]. Morrisette et al. showed that $62 \%$ patients with LBP wore an inextensible LSO, and $78 \%$ patients with LBP wore an extensible LSO daily. Hsieh et al. showed that $65 \%$ of their subjects wore their lumbar support for $>7$ hour/day. These two studies assumed the compliance rate to be the number of subjects that wore their lumbar supports in the manner that was recommended. However, in our study, the compliance rate was determined as the ratio of LSO wearing time monitored by temperature sensors to the recommended LSO wearing time. Studies that evaluated the preventive role of lumbar supports in LBP incidence have shown low compliance rates $[4,5]$. The subjects in these studies 
may have had no LBP experience; therefore, their compliance rate was low. Jellema et al. [8] reported compliance rates according to a questionnaire from $61 \%$ to $81 \%$ from workers who had a history of LBP. The experience of pain could provide an inducement to use the lumbar support to prevent occurrence of LBP.

Pain intensity decreased in parallel with the compliance rate during the 6-week treatment period. Our result is similar to that of studies in which the effectiveness of the lumbar support was evaluated over periods from 3 weeks to 3 months [7-10,24-26]. Some studies showed no significant differences in pain severity with lumbar-support use. Unfortunately, no compliance rate data is available from these studies [27,28], but our study showed an association between compliance rates and pain intensity that could be considered in the assessment of lumbar-support use in future studies.

There is no direct relationship between the functional outcomes, including the disability and fear-avoidance beliefs of subjects and compliance rate during the 6-week treatment. In our study, the disability scale and the fearavoidance behavior of subjects improved after 3 weeks of wearing LSO. Furthermore, the improvement in the behavior of the subjects was consistent at the 1-month followup. Our results are in agreement with literature that evaluated a disability scale in patients with LBP [9-12]. They indicated that the disability score improved when patients wore lumbar supports for periods between 2 weeks and 1 year. Hsieh et al. [12] showed that the functional status of patients with LBP improved only after wearing the lumbar support for 4 weeks. This implies that functional improvement in chronic LBP requires additional time.

Our results indicated an improvement in subjects' fearavoidance behavior during the 6-week treatment. This is inconsistent with the study conducted by Morrisette et al. [9] that revealed no significant difference in fear-avoidance behavior of patients with LBP. This result may be attributed to the fact that they evaluated LSO wearing over a short period of 2 weeks. In the present study, significant improvement in fear-avoidance beliefs was noted after 3 weeks of wearing LSO.

The strength of this study was the utilization of temperature loggers for objectively monitoring LSO wearing. Furthermore, 1-month follow-up after weaning off of LSO was conducted to investigate whether LSO wearing during the 6-week treatment improved (e.g., pain relief) chronic nonspecific LBP.
After descriptive analysis, we found the data had covered a limited range of compliance rates. If we could recruit more subjects, it would have been possible to capture more diverse compliance rates and enrich our conclusions.

\section{Conclusions}

The compliance rate plays an important role in the possible impact of lumbar-support use on patients with chronic nonspecific LBP. We recommend that the effect lumbar-support use should be evaluated objectively in patients with acute and subacute LBP. More investigation is needed on lumbar-support wearing time to derive the optimal wearing time of lumbar supports in chronic LBP management.

\section{Conflict of Interest}

No potential conflict of interest relevant to this article was reported.

\section{ORCID}

Maryam Hekmatfard: https:/orcid.org/0000-0001-7351-9514 Mohammad Ali Sanjari: https://orcid.org/0000-0003-3425-7448 Nader Maroufi: https://orcid.org/0000-0003-0719-367X Hassan Saeedi: https://orcid.org/0000-0001-7066-5081 Esmail Ebrahimi: https://orcid.org/0000-0002-3968-0045 Hamid Behtash: https://orcid.org/0000-0002-6661-4465

\section{References}

1. Andersson GB. Epidemiological features of chronic low-back pain. Lancet 1999;354:581-5.

2. Airaksinen O, Brox JI, Cedraschi C, et al. Chapter 4. European guidelines for the management of chronic nonspecific low back pain. Eur Spine J 2006;15 Suppl 2:S192-300.

3. Turk DC, Rudy TE, Sorkin BA. Neglected topics in chronic pain treatment outcome studies: determination of success. Pain 1993;53:3-16.

4. van Poppel MN, Koes BW, van der Ploeg T, Smid T, Bouter LM. Lumbar supports and education for the prevention of low back pain in industry: a randomized controlled trial. JAMA 1998;279:1789-94.

5. Reddell CR, Congleton JJ, Dale Huchingson R, Montgomery JF. An evaluation of a weightlifting belt 
and back injury prevention training class for airline baggage handlers. Appl Ergon 1992;23:319-29.

6. Jellema P, van Tulder MW, van Poppel MN, Nachemson AL, Bouter LM. Lumbar supports for prevention and treatment of low back pain: a systematic review within the framework of the Cochrane Back Review Group. Spine (Phila Pa 1976) 2001;26:377-86.

7. Calmels P, Queneau P, Hamonet C, et al. Effectiveness of a lumbar belt in subacute low back pain: an open, multicentric, and randomized clinical study. Spine (Phila Pa 1976) 2009;34:215-20.

8. Jellema P, Bierma-Zeinstra SM, Van Poppel MN, Bernsen RM, Koes BW. Feasibility of lumbar supports for home care workers with low back pain. Occup Med (Lond) 2002;52:317-23.

9. Morrisette DC, Cholewicki J, Logan S, Seif G, McGowan S. A randomized clinical trial comparing extensible and inextensible lumbosacral orthoses and standard care alone in the management of lower back pain. Spine (Phila Pa 1976) 2014;39:1733-42.

10. Oleske DM, Lavender SA, Andersson GB, Kwasny MM. Are back supports plus education more effective than education alone in promoting recovery from low back pain?: results from a randomized clinical trial. Spine (Phila Pa 1976) 2007;32:2050-7.

11. Kawchuk GN, Edgecombe TL, Wong AY, Cojocaru A, Prasad N. A non-randomized clinical trial to assess the impact of nonrigid, inelastic corsets on spine function in low back pain participants and asymptomatic controls. Spine J 2015;15:2222-7.

12. Hsieh CY, Phillips RB, Adams AH, Pope MH. Functional outcomes of low back pain: comparison of four treatment groups in a randomized controlled trial. J Manipulative Physiol Ther 1992;15:4-9.

13. Nicholson GP, Ferguson-Pell MW, Smith K, Edgar M, Morley T. The objective measurement of spinal orthosis use for the treatment of adolescent idiopathic scoliosis. Spine (Phila Pa 1976) 2003;28:2243-50.

14. Ferreira-Valente MA, Pais-Ribeiro JL, Jensen MP. Validity of four pain intensity rating scales. Pain 2011;152:2399-404.

15. Williamson A, Hoggart B. Pain: a review of three commonly used pain rating scales. J Clin Nurs 2005;14:798-804.

16. Mousavi SJ, Parnianpour M, Mehdian H, Montazeri A, Mobini B. The Oswestry Disability Index, the Roland-Morris Disability Questionnaire, and the
Quebec Back Pain Disability Scale: translation and validation studies of the Iranian versions. Spine (Phila Pa 1976) 2006;31:E454-9.

17. Fairbank JC, Pynsent PB. The Oswestry Disability Index. Spine (Phila Pa 1976) 2000;25:2940-52.

18. Waddell G, Newton M, Henderson I, Somerville D, Main CJ. A Fear-Avoidance Beliefs Questionnaire $(F A B Q)$ and the role of fear-avoidance beliefs in chronic low back pain and disability. Pain 1993;52:157-68.

19. Rostami M, Noorian N, Mansournia MA, Sharafi E, Babaki AE, Kordi R. Validation of the Persian version of the fear avoidance belief questionnaire in patients with low back pain. J Back Musculoskelet Rehabil 2014;27:213-21.

20. Hasler CC, Wietlisbach S, Buchler P. Objective compliance of adolescent girls with idiopathic scoliosis in a dynamic SpineCor brace. J Child Orthop 2010;4:211-8.

21. Helfenstein A, Lankes M, Ohlert K, et al. The objective determination of compliance in treatment of adolescent idiopathic scoliosis with spinal orthoses. Spine (Phila Pa 1976) 2006;31:339-44.

22. Weinstein SL, Dolan LA, Wright JG, Dobbs MB. Effects of bracing in adolescents with idiopathic scoliosis. N Engl J Med 2013;369:1512-21.

23. Chalmers E, Lou E, Hill D, Zhao HV. An advanced compliance monitor for patients undergoing brace treatment for idiopathic scoliosis. Med Eng Phys 2015;37:203-9.

24. Hsieh CY, Adams AH, Tobis J, et al. Effectiveness of four conservative treatments for subacute low back pain: a randomized clinical trial. Spine (Phila $\mathrm{Pa}$ 1976) 2002;27:1142-8.

25. Million R, Nilsen KH, Jayson MI, Baker RD. Evaluation of low back pain and assessment of lumbar corsets with and without back supports. Ann Rheum Dis 1981;40:449-54.

26. Penrose KW, Chook K, Stump JL. Acute and chronic effects of pneumatic lumbar support on muscular strength, flexibility, and functional impairment index. Sports Med Train Rehab 1991;2:121-9.

27. Doran DM, Newell DJ. Manipulation in treatment of low back pain: a multicentre study. Br Med J 1975;2:161-4.

28. Coxhead CE, Inskip H, Meade TW, North WR, Troup JD. Multicentre trial of physiotherapy in the management of sciatic symptoms. Lancet 1981;1:1065-8. 